\title{
Unstable odontoid fractures: technical appraisal of anterior extrapharyangeal open reduction internal fixation for irreducible unstable odontoid fractures. Patient series
}

\author{
Sushil Patkar, MCh \\ Department of Neurosurgery, Poona Hospital \& Research Centre, Maharashtra, India
}

BACKGROUND Displaced odontoid fractures that are irreducible with traction and have cervicomedullary compression by the displaced distal fracture fragment or deformity caused by facetal malalignment require early realignment and stabilization. Realignment with ultimate solid fracture fusion and atlantoaxial joint fusion, in some situations, are the aims of surgery. Fifteen such patients were treated with direct anterior extrapharyngeal open reduction and realignment of displaced fracture fragments with realignment of the atlantoaxial facets, followed by a variable screw placement (VSP) plate in compression mode across the fracture or anterior atlantoaxial fixation (transarticular screws or atlantoaxial plate screw construct) or both.

OBSERVATIONS Anatomical realignment with rigid fixation was achieved in all patients. Fracture fusion without implant failure was observed in $100 \%$ of the patients at 6 months, with 1 unrelated mortality. Minimum follow-up has been 6 months in 14 patients and a maximum of 3 years in 4 patients, with 1 unrelated mortality.

LESSONS Most irreducible unstable odontoid fractures can be anatomically realigned by anterior extrapharyngeal approach by facet joint manipulation. Plate (VSP) and screws permit rigid fixation in compression mode with $100 \%$ fusion. Any associated atlantoaxial instability can be treated from the same exposure.

https://thejns.org/doi/abs/10.3171/CASE21501

KEYWORDS unstable odontoid fractures; atlantoaxial dislocation; atlantoaxial fixation; atlantoaxial instability; odontoid fracture

Fracture displacement and irreducibility is multifactorial following odontoid trauma. Irreducible unstable odontoid fractures are more common in young patients with high-impact and high-velocity trauma when strong disruptive forces act upon the osseoligamentous complex at the base of the skull. These may be associated with significant cord injury and associated trauma (brain, viscera, and long bones). Following diagnosis, immediate attempt to realign the bones with skull traction is a standard procedure. The absence of standard guidelines about traction and a small but certain chance of devastating deterioration following traction are causes of concern, and most surgeons would plan early surgical realignment and stabilization. Transoral decompression followed by posterior stabilization has been the main option. Posterior manipulation and realignment with fixation in properly selected cases has been described recently.
Open reduction internal fixation by a single-stage unilateral direct anterior extrapharyngeal approach may be another option for successful fracture realignment and atlantoaxial fixation resulting in solid bony fusion in unstable, irreducible odontoid fractures.

\section{Study Description \\ Methods}

Between February 2011 and December 2020, we surgically treated 15 patients with unstable odontoid fractures with irreducible atlantoaxial dislocation. Fractures were considered irreducible after they remained displaced and irreducible in spite of a 3-day trial of skull traction up to 12 $\mathrm{kg}$. Mechanisms of trauma included motorcycle accidents (9 patients), high-speed car accidents (2 patients), diving (2 patients), and falls from

ABBREVIATIONS 3D = three dimensional; ASOF = anterior odontoid screw fixation; $C T$ = computerized axial tomography; MRI = magnetic resonance imaging; PAFF = posterior atlantoaxial fixation fusion; VSP = variable screw placement. INCLUDE WHEN CITING Published November 15, 2021; DOI: 10.3171/CASE21501.

SUBMITTED September 22, 2021. ACCEPTED September 27, 2021.

(C) 2021 The authors, CC BY-NC-ND 4.0 (http://creativecommons.org/licenses/by-nc-nd/4.0/). 


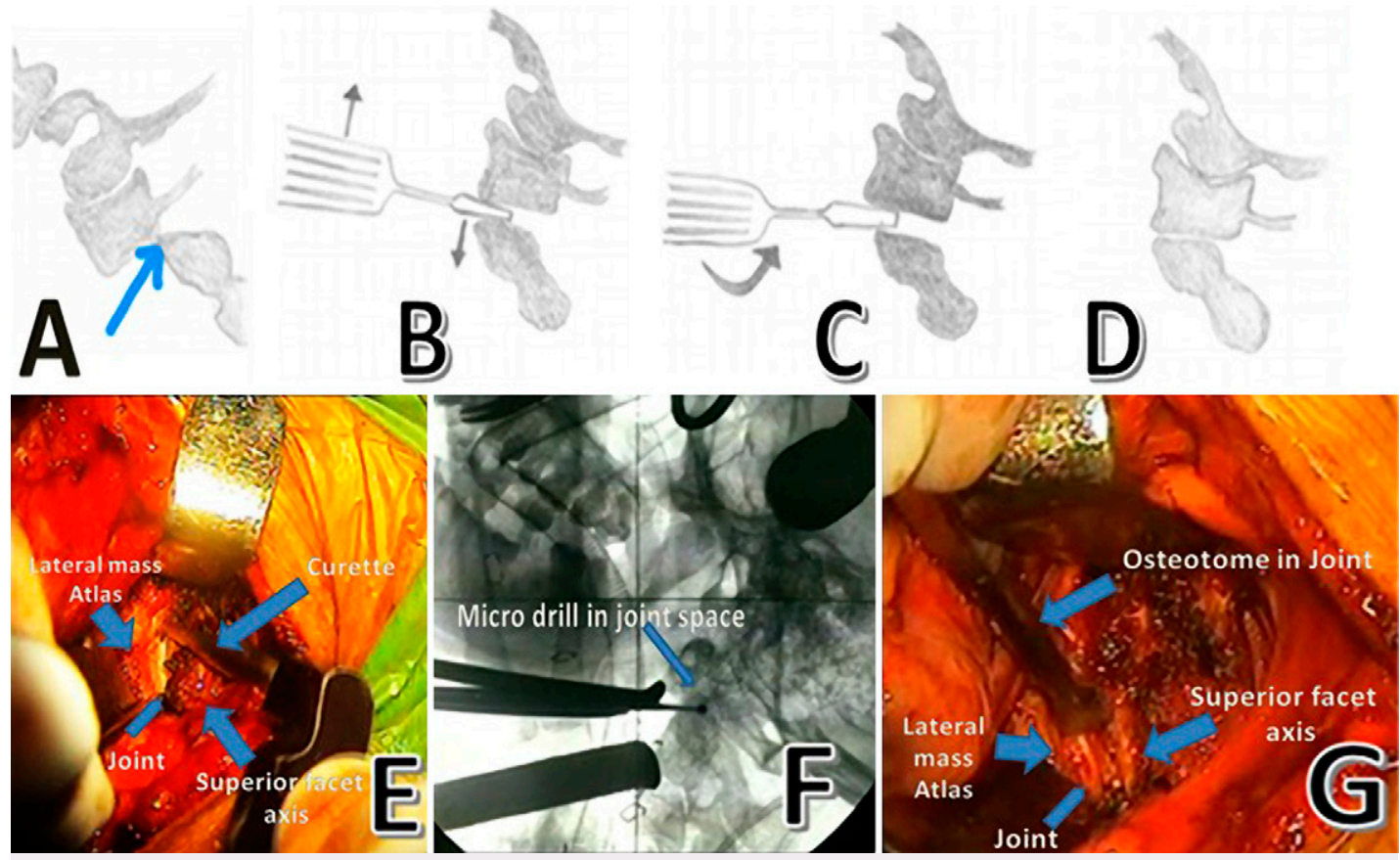

FIG. 1. A: Identifying the joint space below the lateral mass of the atlas (anatomical drawing). B: Inserting osteotome for manipulation (anatomical drawing). C: Rotating the osteotome to open the joint and push and realign the atlas upward and backward (anatomical drawing). D: Repositioned and realigned facet joint (anatomical drawing). E: Intraoperative identification of joint space with a microcurette below the anteriorly and inferiorly displaced lateral mass of atlas over the superior facet of the axis. F: Intraoperative fluoroscopic image of microdrill in atlantoaxial joint space. G: Intraoperative image of osteotome in atlantoaxial joint space to manipulate and realign the atlantoaxial facets.

high heights (2 patients). Most were males (13 males/2 females; ages: 21-64 years), and 13 patients were less than 40 years old. Twelve patients underwent surgery within 3 days of injury, and the remaining 3 patients underwent surgery within 10 days of injury. Skull traction (Gardner-Wells tongs) was applied in all cases, and a trial of 3 days was given to 5 patients. Over the years, we realized the success of the procedure to realign almost all fractures and started taking patients earlier for surgery. None had realigned before surgery.

Apart from routine investigations for fitness for surgery, all patients underwent lateral radiographs for diagnosis and reducibility after traction and computerized axial tomography (CT) scanning with bone window settings and three-dimensional (3D) reconstruction to study the displacement of the fracture fragment and the facetal malalignment (atlantoaxial dislocation), other fractures, and bone stock for length of screws required. Magnetic resonance imaging (MRI) was performed to study cord injury, and magnetic resonance angiography was performed for vertebral artery position and associated spinal cord trauma.

Informed consent for surgery was obtained from all patients and next of kin. Institutional review board approval was not necessary, as surgical treatment is the standard of care for most unstable odontoid fractures. The surgical approach and implants have been used before, and the results have been extensively published in the literature.

\section{Operative Technique}

Following general anesthesia and fiber optic intubation, the patient was positioned supine on a radiolucent table compatible for intraoperative radiography. The head was fixed with adhesive tape in mild extension with a minimal tilt to the left side sparing the skull traction without weights (Gardner-Wells) applied earlier, so that it could be available for manipulation intraoperatively. A unilateral right-side, submandibular extrapharyngeal approach was used to reach the fracture site where fracture hematoma could be identified. The anterior longitudinal ligament was divided, and longus coli muscles were dissected away from midline to expose the anterior surface of the arch of atlas, odontoid process, body of axis. The lateral masses of the atlas, atlantoaxial joints, and the superior facets of the axis were exposed bilaterally, which sometimes required division of the longus coli and longus capitis up to the lateral border of the joints; removal of soft tissue and synovial joint between the anterior arch of the atlas and odontoid (which may require drilling of the inferior half of the rim of the atlas) and opening the atlantoaxial facet joint capsule generously on both sides aided the loosening of the deformity. The fracture site was opened and manipulated into correct alignment (both anterior facet edges of the atlas and axis were in the same sagittal and coronal planes) using the skull traction for manipulation under " $C$ " arm guidance.

\section{Reduction Techniques}

In anterior displacements, distracting the atlantoaxial joints using a 5-mm osteotome passed into the joint for $1 \mathrm{~cm}$ and tilted to lever the lateral mass of the atlas upward and backward resulted in reduction and realignment (Fig. 1).

In posterior or coronal displacements, drilling a 3-mm hole and securing a tap for 5-8 $\mathrm{mm}$ (depending upon thickness of bone seen on preoperative CT) into the distal fragment or anterior tubercle of the atlas permitted manipulation for achieving the correct anatomical position of 


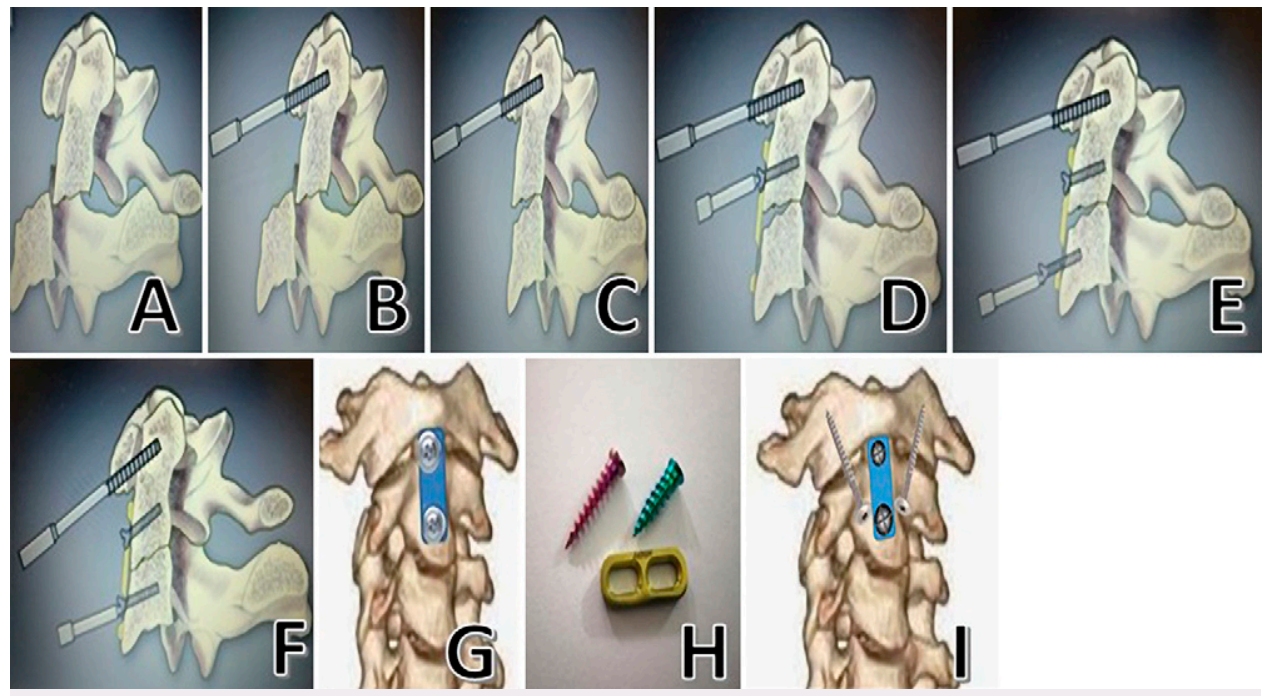

FIG. 2. A: Posteriorly displaced impacted fracture (anatomical image). B: Impaling the anterior arch of the atlas and distal fracture fragment with a tap (anatomical image). C: Repositioning the distal fragment (anatomical image). D: VSP plate fixed, proximal screw in distal fragment (anatomical image). E: Proximal screw passed close to screw hole (anatomical image). F: Tightening the proximal screw results in compression of fracture site (anatomical image). G: Position of VSP plate and screw construct (anatomical image). H: Customized VSP plate and screws by Jayon implants, Pvt. Ltd. I: Bilateral anterior transarticular screws for associated atlantoaxial instability (anatomical image).

the facet joints. The skull tongs can be manipulated simultaneously by an assistant for distraction and to realign the facets of the atlas and axis (Fig. 2).

\section{Odontoid Fracture Fixation}

A customized titanium VSP plate (Jayon implants, Pvt. Ltd) of $3 \mathrm{~mm}$ thickness with $3.5-\mathrm{mm}$ screws (18-22 $\mathrm{mm}$ according to preoperative CT scan measurements) was fixed into the distal fragment. Another hole for the proximal body was drilled through the plate under radiography control, close to the near edge of the proximal hole in the plate, and a $3.5-\mathrm{mm}$ screw (length according to preoperative CT scan) was passed, which on tightening (due to the sloping edges of the screw holes), migrated toward the center of the screw hole, resulting in reduction and compression at the fracture (Video 1).

VIDEO 1. Clip showing animation of anterior open reduction and internal fixation (VSP plate screw construct) for unstable odontoid fractures. Click here to view.

\section{Joint Gardening and Grafting}

The joint surfaces were abraded using angled curettes and angled 3-mm microburr until there was blood oozing from the endplates. Cortical and cancellous bone graft harvested from the iliac crest was packed snuggly into the joint.

\section{Atlantoaxial Joint Fixation}

Anterior Atlantoaxial Transarticular Screws

The subfacetal surface of the C2 was cleared of soft tissue bilaterally. The anterior surface of the $\mathrm{C} 2$ body was made flat with a diamond 4-mm drill. The $\mathrm{C} 1$ lateral mass was cleared of soft tissue until its upper lateral limit could be visualized bilaterally. The skull tongs were used to correct rotation and achieve satisfactory reduction of the atlantoaxial dislocation by extension, which was confirmed by intraoperative radiography. The midpoint of the $\mathrm{C} 2$ lateral mass was identified, and the entry point chosen was $5 \mathrm{~mm}$ inferomedial to the midpoint, and with a $3.5-\mathrm{mm}$ drill bit, a hole was drilled directed to the upper outer limit of the $\mathrm{C} 1$ lateral mass with a $25-30^{\circ}$ downward tilt (intraoperative " $\mathrm{C}$ " arm imaging used to confirm trajectory) toward the upper posterior end of the lateral mass. The drill could be seen and felt traversing the joint space bone graft and passing into the lateral mass of C1. A 3.5-mm titanium cortical screw of $20-24 \mathrm{~mm}$ in length was passed to secure the $\mathrm{C} 1-2$ lateral masses. The procedure was repeated on the opposite side.

\section{Anterior Atlantoaxial Screw Plate Fixation}

The anterior surface of the lateral mass of $\mathrm{C} 1$ was cleared of soft tissue. At $5 \mathrm{~mm}$ above the midpoint of the $\mathrm{C} 1-2$ joint, a hole was drilled with a 3-mm drill bit directed $20^{\circ}$ upward and outward (direction and length based on preoperative CT scan analysis) to a depth of $15-20 \mathrm{~mm}$; 4-mm-thick cortical titanium screws of $18-20 \mathrm{~mm}$ in length were passed through the customized $3.5-\mathrm{mm}$ VSP plate of adequate length, and the lower hole was adjusted over the anterior surface of the C2 body just above the C2-3 disk space; the drill hole was made with the plate in position directed $20^{\circ}$ medially and to reach the posterior cortex according to calculations on the preoperative CT scan, usually $18-20 \mathrm{~mm}$; and another 4-mm cortical screw was passed to secure the plate. Before fixing the lower screw, the head was extended, rotation-corrected, and positioned to reduce the atlantoaxial dislocation, which was confirmed with intraoperative " $\mathrm{C}$ " arm imaging. The procedure was repeated on the opposite side. 


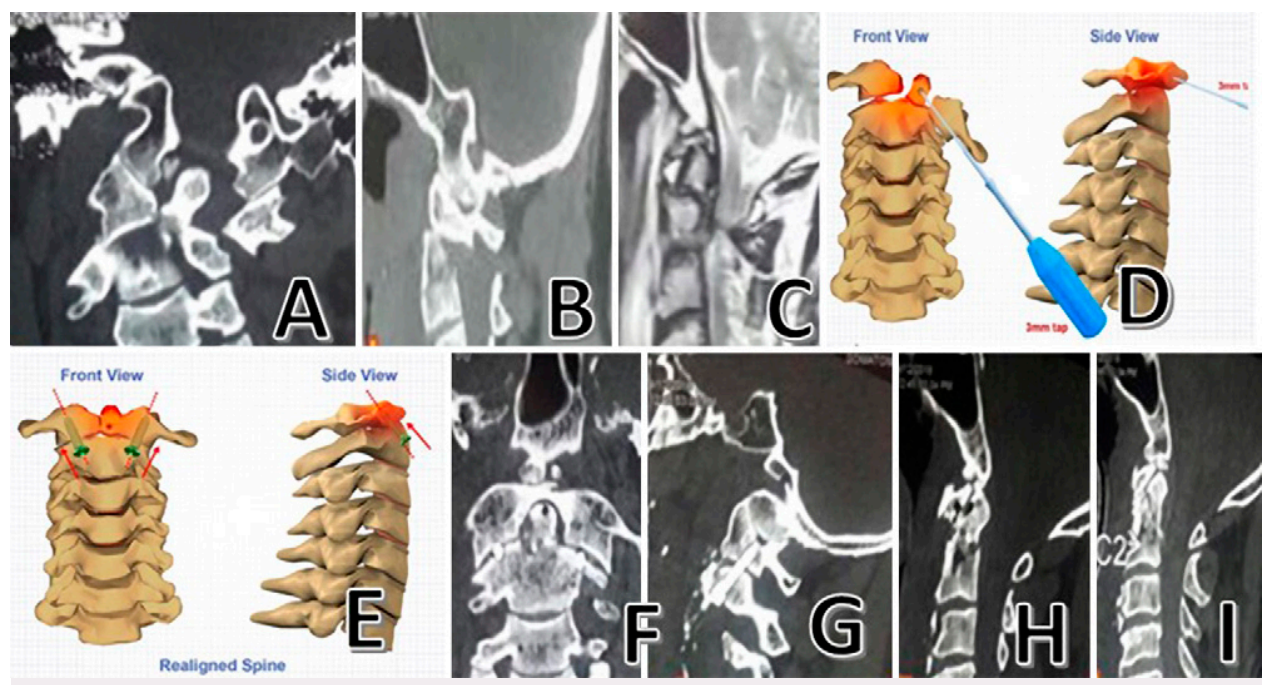

FIG. 3. A: Fracture odontoid with coronal displacement and locked atlantoaxial facet. B: Sagittal CT scan of displaced lateral mass. C: Sagittal MRI showing significant thecal distortion. D: Realignment by impaling the distal odontoid fracture fragment after partial drilling of atlas and repositioning of the odontoid (anatomical image). E: Atlantoaxial fixation with bilateral anterior transarticular screws (anatomical image). F: Coronal CT image showing satisfactory realignment and drilled anterior arch of atlas with hole in odontoid for tap fixation during manipulation for realignment. G: Sagittal reconstruction CT scan showing transarticular screw position. $\mathrm{H}$ : Three months postoperative, coronal midsagittal CT scan reconstruction reveals good realignment and fracture fusion. I: Six-month coronal midsagittal CT scan reconstruction with almost complete fracture fusion.

Intraoperative dynamic imaging using "C" arm was done to confirm rigidity of the fixation. All wounds were closed with a retropharyngeal drain, and patients were mobilized the next day with a hard collar to be used for 4 weeks.

Follow-up dynamic radiographs were taken before discharge from the hospital and every 3-6 months thereafter until good fracture fusion was seen. Some patients who were willing were imaged up to 3 years for academic purposes (Figs. 3-5, Video 2).

VIDEO 2. Clip showing postoperative neck movements after open reduction internal fixation and anterior fixation for odontoid fracture. Click here to view.

\section{Results}

Four patients with preoperative quadriparesis with tingling numbness and sensory loss in all limbs recovered over 3 months and could perform activities of daily living. Three had associated brain injuries and prolonged loss of consciousness with associated polytrauma; these patients could not be assessed adequately and underwent surgery after stabilization and treatment of other trauma; they required tracheostomy and Ryles tube feeding for 4 weeks and were eventually discharged with significant disability that necessitated use of a wheelchair and constant care for activities of daily living. Two patients had postoperative transient right hypoglossal weakness for 3-12 days, 3 had persistent postoperative neck and occipital pain for 2 months, which required analgesics. One patient had preoperative dysphagia after the injury and also developed hypoglossal weakness, which worsened the dysphagia and required Ryles tube feeding for 2 weeks, but had a complete recovery. One patient died 3 weeks after surgery due to acute myocardial infarction, unrelated to surgery. No patient needed intraoperative transfusion, and no surgery lasted more than 180 minutes. Except for the unrelated mortality, anatomical realignment of the fracture with fusion without implant failure was observed in $100 \%$ of the patients at 6 months. Minimum follow-up has been 6 months in 14 patients and a maximum of 3 years in 4 patients.

\section{Discussion \\ Observations}

The popular Anderson and D'Alonzo classification of odontoid fractures does not include displacement, instability, irreducibility, or associated fractures, which have additional therapeutic implications. ${ }^{1}$ Unstable odontoid fractures are those in which weightbearing can result in spinal cord compression by the atlantoaxial dislocation or the displaced fracture fragment. Some complex unstable odontoid fractures are displaced, impacted with deformity, which may be irreducible with preoperative skull traction. There are no established guidelines for the amount of weight or duration for preoperative skull traction in complex unstable odontoid fractures, and traction can occasionally be disastrous. ${ }^{2}$ These require early surgical realignment and stabilization. ${ }^{3}$

Unstable displaced odontoid fractures result from severe hyperflexion or hyperextension forces, which cause the fracture and additional injury of the longitudinal part of the cruciate ligament but preserved transverse ligament and resultant displacement of the distal fragment of the odontoid, along with the atlas, anteriorly or posteriorly with facetal malalignment. ${ }^{4}$ In most displaced odontoid fractures, the atlantodental interval is preserved even in those with significant anteroposterior displacement or angulation of the distal fragment, implying that the transverse alar ligament is preserved and the vertical component of the cruciate ligament is disrupted at 


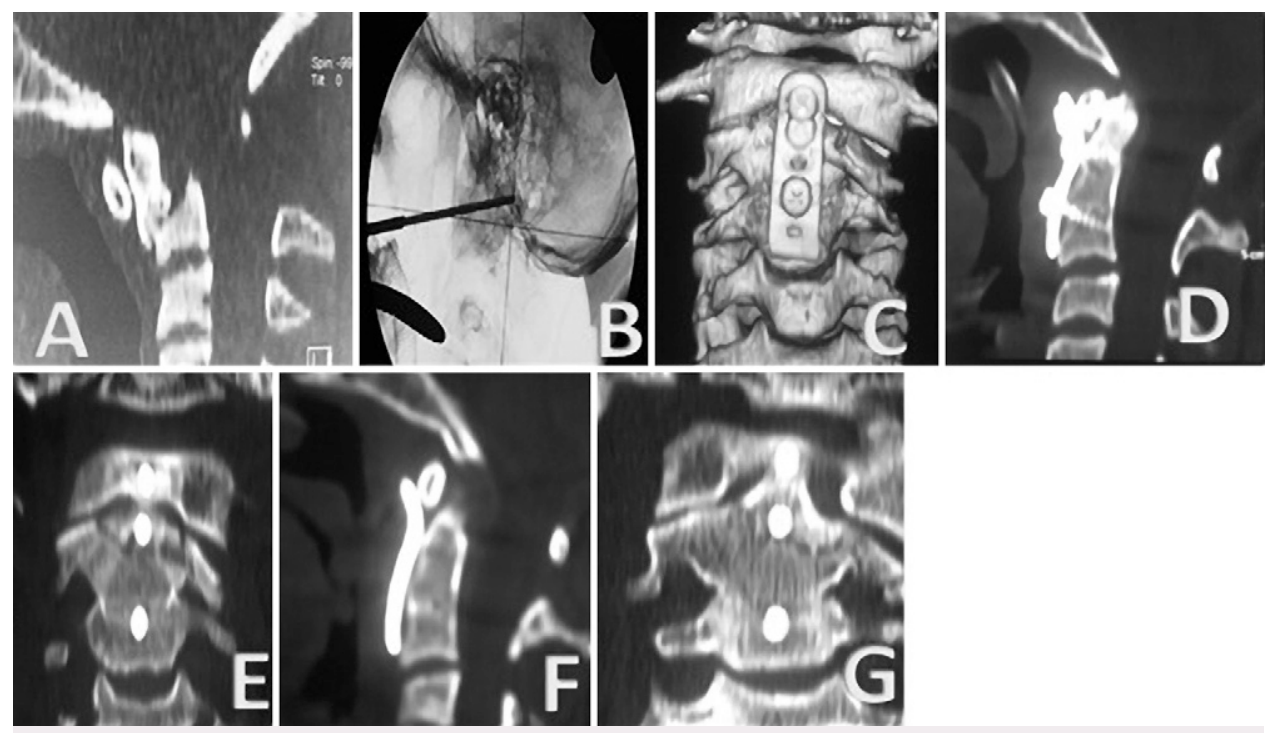

FIG. 4. A: Anterioinferiorly displaced and impacted odontoid fracture. B: Anterior arch of atlas and tip of odontoid impaled with a tap after drilling and fragment realigned. C: CT scan 3D image showing customized titanium plate fixed to hold anterior arch with odontoid tip, distal fracture fragment, and proximal fragment. D: Sagittal CT scan reconstruction at 3 months showing fracture healing with satisfactory implant position. E: Postoperative coronal CT scan reconstruction at 3 months showing healing fracture. F: Postoperative sagittal CT scan reconstruction at 12 months showing fused and remodeled odontoid. G: Postoperative coronal CT scan at 12 months showing fused fracture gap.

its lower attachment and can be missed in routine MRI and CT imaging. ${ }^{5}$ The extent of instability in odontoid fractures is a function of loss of facetal relationship best seen on parasagittal CT scanning at the midsagittal level of both the joints with $3 \mathrm{D}$ reconstruction of the atlantoaxial complex. ${ }^{6}$ Irreducibility in these displaced fractures is multifactorial. $^{7}$

Anterior odontoid screw fixation (ASOF), the commonest technique used for odontoid fracture fixation, is contraindicated in unstable irreducible misaligned situations. ${ }^{8}$ Transoral reduction followed by ASOF from separate incision and followed by posterior atlantoaxial fixation fusion (PAFF) has been described for such situations; transoral odontoidectomy followed by PAFF can be an option in irreducible displaced unstable odontoid fractures. ${ }^{9,10}$ Anterior odontoid screw with bilateral anterior transarticular screws and anterior plate screw fixation have been described earlier as salvage options. $^{11,12}$

PAFF by the Magerl/Goel-Harms technique has been recommended for unstable fractures. ${ }^{13-15}$ The displaced impacted odontoid fracture fragment can be reduced by complex posterior facet distraction and manipulation. ${ }^{16}$ A low-profile VSP plates and screws construct can simplify realignment, manipulation, and compression during PAFF. ${ }^{17}$ However, vertebral artery injury remains a potentially serious problem with PAFF, in addition to the necessity for $\mathrm{C} 2$ root division. Preoperative vertebral angiography, 3D printing, intraoperative navigation, and using " $\mathrm{O}$ " arm are recommended in literature to enhance safety. ${ }^{18}$ The posterior surgery disrupts the posterior tension band permanently, and the high-profile polyaxial screw rod complex prevents muscles from reattachment to the posterior craniocervical bones permanently. ${ }^{19}$

The anterior approach for atlantoaxial stabilization has been described in literature. ${ }^{20-23}$ The anterior extrapharyngeal VSP plate screw fixation for odontoid fractures can be performed in most cases (more than 95\%) when, on extension, the angles of the mandible are at or above the C2-3 disc space on a lateral radiograph. ${ }^{24,25}$ The unilateral anterior extrapharyngeal approach to both the atlantoaxial joints is without any risk to the vertebral arteries and preserves the $\mathrm{C} 2$ root. This approach in supine position of the neck seems to be safer in highly unstable fractures. The exposure between muscle planes with almost negligible muscle detachment (longus coli) seems biomechanically superior. After exposure of the fracture and both the atlantoaxial facets, additional loosening of the atlantoaxial complex by generous opening of the joint capsules, division of the longus coli and longus capitis can unlock most fresh fractures. Joint manipulation with an osteotome or impaling the anterior arch of the atlas with the odontoid tip using a 3.5-mm tap permits safe manipulation and reduction of the displaced distal fragment, along with the atlantoaxial facets under vision assisted by skull-tong manipulation. The VSP principle relies on fracture-site compression for enhancing fusion with minimal callus. ${ }^{26-28}$

This approach may permit safe and wide exposure of both the facet joints for endplate abrasion and corticocancellous bone grafting along the natural line of weight transmission. This approach exposes a wide area of the lateral mass and the body of the axis, permitting safe rigid fixation using either transarticular screws or a plate screw construct under vision without the need for navigation. If required, C2-3 fusion-fixation can be easily added for associated unstable Hangman's fracture. ${ }^{29}$ Transoral excision of odontoid for displaced impacted odontoid fractures can be avoided by this technique; however, it can be easily performed if required by this approach. The weight of the skull is transmitted by the condyles to the lateral masses of the atlas and most of it onto the superior facets and body of the axis to the subaxial spine; some weight is 


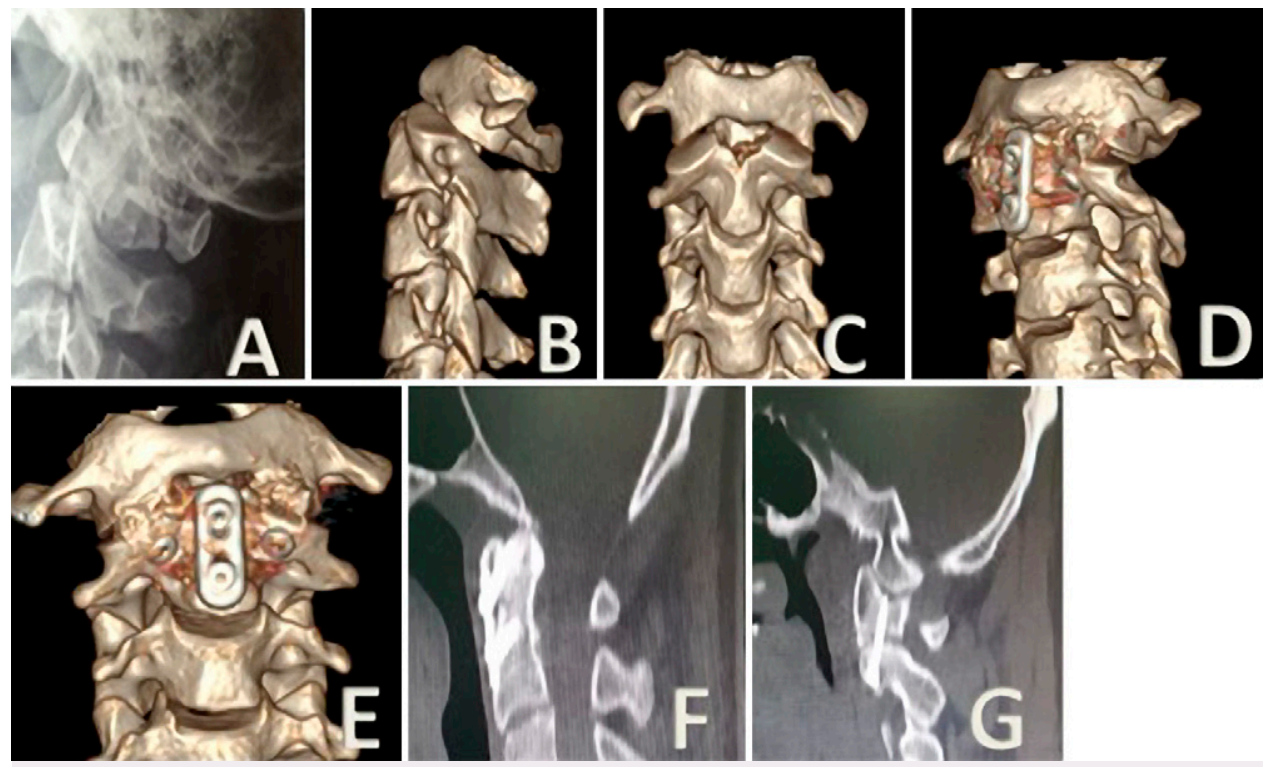

FIG. 5. A: Lateral radiograph: fracture odontoid with posterior displacement of atlas over axis. B: Sagittal CT scan 3D reconstruction showing posteriorly displaced atlas with facetal malalignment. C: Coronal CT scan $3 \mathrm{D}$ reconstruction showing fracture odontoid with posterior displacement of atlas over axis and facetal malalignment. D: Postoperative oblique CT scan 3D reconstruction showing implant construct with bone grafts in atlantoaxial joints and realigned facets. E: Postoperative coronal CT scan 3D reconstruction at 6 months showing implant construct with bone grafts in atlantoaxial joints and realigned facets. F: Postoperative sagittal CT scan reconstruction at 6 months completely fused and remodeled odontoid. G: Postoperative sagittal CT scan reconstruction at 6 months showing realigned facets, transarticular screw, and fusion across the joint.

transferred onto the posterior elements of the axis onto the lateral masses of the subaxial spine. ${ }^{30}$

\section{Lessons}

Unilateral, extrapharyngeal approach permits open reduction and direct fixation of the odontoid fracture in compression mode. The associated atlantoaxial instability can be rigidly fixed and adequately grafted for fusion, simultaneously, from the same exposure along the line of natural weight transmission without disturbing the posterior tension band.

The anterior technique seems simple, safe, and feasible in most cases of irreducible unstable odontoid fractures, with acceptable complications, and permits 100\% fracture realignment and fusion. The patients in this group were younger than in other published series, and more cases must be reported before long-term complications of the procedure and implants become apparent. This technique has the potential to become the procedure of choice in most cases of unstable irreducible odontoid fractures.

\section{Acknowledgments}

I acknowledge Dr. Pradnya Patkar from the Royal Preston Hospital, United Kingdom, for editing, literature review, and illustrations.

\section{References}

1. Anderson LD, D'Alonzo RT. Fractures of the odontoid process of the axis. J Bone Joint Surg Am. 1974;56(8):1663-1674.

2. Lewallen RP, Morrey BF, Cabanela ME. Respiratory arrest following posteriorly displaced odontoid fractures. Case reports and review of the literature. Clin Orthop Relat Res. 1984;(188):187-190.
3. Steltzlen C, Lazennec JY, Catonné Y, Rousseau MA. Unstable odontoid fracture: surgical strategy in a 22-case series, and literature review. Orthop Traumatol Surg Res. 2013;99(5):615-623.

4. Clark $C R$, White AA 3rd. Fractures of the dens. A multicenter study. J Bone Joint Surg Am. 1985;67(9):1340-1348.

5. Nidecker AE, Shen PY. Magnetic resonance imaging of the craniovertebral junction ligaments: normal anatomy and traumatic injury. J Neurol Surg B Skull Base. 2016;77(5):388-395.

6. Chandra PS, Goyal N, Chauhan A, Ansari A, Sharma BS, Garg A. The severity of basilar invagination and atlantoaxial dislocation correlates with sagittal joint inclination, coronal joint inclination, and craniocervical tilt: a description of new indexes for the craniovertebral junction. Neurosurgery. 2014;10(4)(suppl 4):621-630.

7. Salunke P, Sahoo SK. Irreducible posterior C1-2 dislocation with retrolisthesis of dens: hindsight from 10 cases. World Neurosurg. 2019;123:e202-e210.

8. Lee TK, Han MS, Lee SK, Moon BJ, Lee JK. Outcomes of patients undergoing anterior screw fixation for odontoid fracture and analysis of the predictive factors for surgical failure. Neurospine. 2020;17(3):603-609.

9. Wang T, Zeng B, Xu J. Transoral reduction of irreducible posteriorly displaced odontoid fracture. Eur Spine J. 2011;20(Suppl 2):S227-S230.

10. Kirankumar MV, Behari S, Salunke P, Banerii D, Chhabra DK, Jain VK. Surgical management of remote, isolated type II odontoid fractures with atlantoaxial dislocation causing cervical compressive myelopathy. Neurosurgery. 2005;56(5):1004-1012.

11. Vaccaro AR, Madigan L, Ehrler DM. Contemporary management of adult cervical odontoid fractures. Orthopedics. 2000;23(10):1109-1115.

12. Platzer P, Thalhammer G, Krumboeck A, Schuster R, KutschaLissberg F, Zehetgruber I, et al. Plate fixation of odontoid fractures without C1-C2 arthrodesis: practice of a novel surgical technique for stabilization of odontoid fractures, including the opportunity to extend the fixation to C3. Neurosurgery. 2009;64(4):726-733. 
13. Goel A, Laheri V. Plate and screw fixation for atlanto-axial subluxation. Acta Neurochir (Wien). 1994;129(1-2):47-53.

14. Magerl $F$, Seemann PS. Stable posterior fusion of the atlas and axis by transarticular screw fixation. In: Kehr P, Weidner A, eds. Cervical Spine I. Vol. 1. Springer; 1987:322-327.

15. Buchmann N, Schweizer C, Kirschke JS, Rienmüller A, Gempt J, Ringel $F$, et al. C1-C2 posterior screw fixation in atlantoaxial fractures revisited: technical update based on 127 cases. Eur Spine J. 2020;29(5):1036-1042.

16. Salunke P, Sahoo SK, Savardekar A, Ghuman M, Khandelwal NK. Factors influencing feasibility of direct posterior reduction in irreducible traumatic atlantoaxial dislocation secondary to isolated odontoid fracture. Br J Neurosurg. 2015;29(4):513-519.

17. Patkar S. Posterior atlantoaxial fixation with new subfacetal axis screw trajectory avoiding vertebral artery with customized variable screw placement plate and screws to enhance biomechanics of fixation. Neurosurg Focus Video. 2020;3(1):V10.

18. Harel R, Nulman M, Knoller N. Intraoperative imaging and navigation for C1-C2 posterior fusion. Surg Neurol Int. 2019;10:149.

19. Nemani V, Kim HJ. The Management of Unstable Cervical Spine Injuries. Clin Med Insights Trauma Intensive Med. 2014;5:7-10.

20. Koller H, Kammermeier V, Ulbricht D, Assuncao A, Karolus S, van den Berg B, Holz U. Anterior retropharyngeal fixation C1-2 for stabilization of atlantoaxial instabilities: study of feasibility, technical description and preliminary results. Eur Spine J. 2006;15(9):1326-1338.

21. Patkar S. Anterior extra pharyngeal approach and fixation of the atlanto-axial joint with screw plate construct or anterior transarticular screws: and review of literature. Spinal Surg. 2013;27:230-237.

22. Patkar S. Anterior retropharyngeal cage distraction and fixation for basilar invagination: "the wedge technique". Neurospine. 2019; 16(2):286-292.

23. Patkar S. Anterior retropharyngeal cage distraction with atlantoaxial plate-screw fixation for basilar invagination with atlantoaxial dislocation. Neurosurg Focus Video. 2020;3(1):V11.

24. Patkar S. Anterior retropharyngeal plate screw fixation with bilateral anterior transarticular screws for odontoid fractures ... a new comprehensive technique. Neurol Res. 2017;39(7): 581-586.

25. Patkar S. Anterior screw plate fixation for odontoid fractures: a new technique. Neurosurgery. 2017;64(Suppl 1):274.

26. Bagby GW, Janes JM. The effect of compression on the rate of fracture healing using a special plate. Am J Surg. 1958;95(5):761-771.

27. Uhthoff HK, Poitras P, Backman DS. Internal plate fixation of fractures: short history and recent developments. J Orthop Sci. 2006;11(2):118-126.

28. Igna C, Schuszler L. Current concepts of internal plate fixation of fractures. Bull Univ Agric Sci Vet Med Cluj Napoca. 2010;67(2): 118-124.

29. Wang L, Liu C, Zhao QH, Tian JW. Outcomes of surgery for unstable odontoid fractures combined with instability of adjacent segments. J Orthop Surg Res. 2014;9:64-69.

30. Pal GP, Routal RV. A study of weight transmission through the cervical and upper thoracic regions of the vertebral column in man. J Anat. 1986;148:245-261.

\section{Disclosures}

The author reports no conflict of interest concerning the materials or methods used in this study or the findings specified in this paper.

\section{Supplemental Information \\ Videos}

Video 1. https://vimeo.com/617031497.

Video 2. https://vimeo.com/617035527.

Online-Only Content

Supplemental material is available with the online version of the article. Supplemental Fig. 1. https://thejns.org/doi/suppl/10.3171/ CASE21501.

\section{Correspondence}

Sushil Patkar: Poona Hospital \& Research Centre, Maharashtra, India. patneuro@hotmail.com. 